\title{
Influence of Magnetoresistance and Temperature on Permanent Magnet Condition Estimation Methods Using High Frequency Signal Injection
}

\author{
Daniel Fernandez, Maria Martinez, David Reigosa, Juan Manuel Guerrero, Carlos Suares, Fernando Briz \\ Dept. of Elect., Computer \& System Engineering \\ University of Oviedo, Gijón, Spain \\ fernandezalodaniel@uniovi.es, uo224394@uniovi.es,diazdavid@uniovi.es, guerrero@uniovi.es, csuarez@uniovi.es, \\ fernando@isa.uniovi.es
}

\begin{abstract}
Torque production capability of permanent magnet synchronous machines (PMSMs) depends on the permanent magnets (PM) magnetization state (MS). MS is a function of PM initial magnetization level, which decreases as temperature increases. Furthermore, excessive PM temperature can produce irreversible PM demagnetization. PM MS and temperature measurement/estimation is therefore important both for torque control and monitoring purposes. The injection of a high frequency (HF) signal in the stator windings has been shown to be a viable option for temperature and MS estimation. This technique estimates the PM temperature or/and MS from the variation of the stator reflected PM HF resistance. However, since $P M H F$ resistance is affected by both PM temperature and MS due to magnetoresistive effect, separating both effects is not trivial. This paper studies the effect of magnetoresistance and temperature on the PM resistivity, the target being twofold: to understand how temperature and MS estimation methods interfere with each other, and to further use of this knowledge for the development of methods able to estimate the magnet temperature and MS simultaneously ${ }^{1}$
\end{abstract}

Keywords-Permanent Magnet losses, Magnetization State, Magnetoresistance, high frequency signal injection, temperature coefficient of resistivity.

\section{INTRODUCTION}

PMSMs are widely used in the industry due to their high power density, high efficiency and good dynamic response [1]-[2]. One concern with PMSMs is that the PM can be irreversible demagnetized due to a combination of electrical and thermal stresses [1]-[3]. PMs are usually the weakest component of a PMSM in terms of maximum operating temperature, and will set therefore the temperature limit of

${ }^{1}$ This work was supported in part by Spanish Ministry of Education, Culture and Sports through "José Castillejo Program" under grant PX15/00354, by Regional Ministry of Education, Culture and Sport of the Principality of Asturias through "Severo Ochoa Program" under Grant BP13067 and by the Research, Technological Development and Innovation Programs of the Spanish Ministry Economy and Competitiveness, under grant MINECO-17-ENE2016-80047-R. the machine. This is especially true for NdFeB magnets, which show the lowest maximum working temperature, see Table I. The dependency of PMs remanent flux $\mathrm{B}_{\mathrm{r}}$ (see Table I) with temperature [1]-[2], is given by the coefficient $\alpha_{B}$; It can be observed that, for PMs typically used in PMSMs (i.e. Ferrite, AlNiCo, SmCo and $\mathrm{NdFeB}$ ), $\mathrm{B}_{\mathrm{r}}$ decreases as the temperature increases, which adversely impacts the machine torque capabilities. Variations of $\mathrm{B}_{\mathrm{r}}$ with temperature are reversible provided that the maximum working temperature of the magnet ( $\mathrm{T}_{\max }$ in Table $\mathrm{I}$ ) is not surpassed; this is commonly known as temporary demagnetization. Surpassing the maximum working temperature will result in irreversible demagnetization and consequently in an irreversible torque capability loss. Measuring or alternatively estimation of the magnet temperature and/or MS is therefore desirable both for torque control and monitoring purposes.

Measuring PMs temperature or MS in a PMSM is not easy. Direct measurement by means of sensors require modifications of the machine, which places robustness and cost concerns as it requires additional hardware [4]-[5]. To the best of authors' knowledge, up to date, direct PM temperature and field measurement has been limited to laboratory prototypes.

TABLE I. THERMAL CHARACTERYSTICS OF TYPICAL PM MATERIALS

\begin{tabular}{|l|c|c|c|c|}
\hline OF TYPICAL PM MATERIALS \\
\hline PM material & $\alpha_{\mathrm{B}}\left(\% /{ }^{\circ} \mathrm{C}\right)$ & $\mathrm{B}_{\mathrm{r}}(\mathrm{T})$ & $\alpha_{\mathrm{H}}\left(\% /{ }^{\circ} \mathrm{C}\right)$ & $\mathrm{T}_{\max }\left({ }^{\circ} \mathrm{C}\right)$ \\
\hline AlNiCo cast & -0.01 to -0.035 & $0.53-1.38$ & -0.03 & 500 \\
\hline Ferrite & -0.20 & $0.34-0.44$ & +0.3 & 250 \\
\hline SmCo 32L & -0.035 to -0.045 & $1.10-1.15$ & -0.25 & 250 \\
\hline NdFeB52M & -0.1 to -0.12 & $1.43-1.46$ & -0.58 to -0.72 & 90 \\
\hline
\end{tabular}

*Source: [6]

Alternatively to direct measurement, PM temperature and field can be estimated. PM temperature estimation methods can be roughly classified into thermal models [7]-[8], backEMF based methods [8] and signal injection methods [10][12]. Thermal models require knowledge of stator and rotor geometry, materials and cooling system, which makes them highly dependent on the machine design. Back-EMF based methods estimate the magnet temperature from the rotor PM flux linkage, which is estimated from the machine terminal voltages and currents. Back-EMF based methods cannot be 
used at zero and very low speed due to the direct relationship between back-EMF and speed. In addition, although obtaining the PM flux linkage when the machine is rotating at no load is relatively simple, its estimation under load conditions becomes not trivial [13]. A different approach to estimate PMs temperature and MS that overcomes some of the aforementioned limitations is by injecting some type of additional signal in the stator of the machine and measuring the machine response. These types of methods include pulse injection [12] and HF signal injection [10]-[11] and [13]. Pulse injection methods [12] estimate $d$-axis inductance $\left(L_{d}\right)$ variations, which depend on the magnetization level of the PMs and therefore of its temperature. HF signal injection methods estimate the PM temperature from the stator reflected PM HF resistance [10]-[11] and [13].

Two different approaches have been proposed for PM field (MS) estimation: use of the Back-EMF [9] and HF signal injection [14]-[15]. Both methods estimate the MS form measurable stator electrical variables [9], [14] and [15]. Back-EMF MS estimation methods estimate the PM MS from the rotor PM flux linkage, suffering therefore from the same limitations as Back-EMF temperature estimation methods. MS can also be estimated from the variation of PM resistivity with its MS due to magnetoresistance (MR). The resistance is estimated by injecting a HF signal in the stator terminals of the machine via inverter [14]-[16], superposed to the fundamental excitation used to produce torque. Since temperature and MS estimation share similar principles, it is important to develop methods to separate their effects and allow simultaneous estimation of both.

This paper analyzes the influence of MS and temperature on PM resistivity. This will be key for the development of PM temperature and MS estimation methods using HF signal injection, as both are based on the estimation of PM's $\mathrm{HF}$ resistance. Special attention will be paid to $\mathrm{NdFeB}$ magnets, as they are the primary option in PMSMs. Analysis of fully magnetized and fully demagnetized $\mathrm{NdFeB}$ magnets will combined to separate the effects of MS and temperature on PM resistivity.

The paper is organized as follows: analysis of the effects of temperature and MS on the PM resistivity is presented in section II. An experimental setup used to evaluate the influence of temperature and MS on PM HF resistance is presented in section III. Single magnet pieces are used in Section III extension of the results to PMSMs is presented in Section IV. Experimental results in a PMSM are provided in Section V. Finally, the conclusions are presented in Section VI.

\section{Temperature And Magnetic Field EfFect ON PM RESISTIVITY}

There are two sources for PM resistivity variations: 1) PMs' temperature; 2) external fields applied to the PMs, e.g. due to the current flowing through the stator windings. Both effects are discussed following:

\section{A. PMs' resistivity vs. temperature}

Hard magnetic materials are made of metals, i.e. $\mathrm{Nd}, \mathrm{Fe}$, $\mathrm{B}, \mathrm{Sm}, \mathrm{Co}, \mathrm{Al}, \mathrm{Ni} . .$. , their resistivity changing almost linearly with temperature [17]. The degree of change of the electrical resistivity of a PM vs. its temperature is known as temperature coefficient of resistivity $\alpha(1)$, where $\rho\left(T_{0}\right)$ is the resistivity at the reference temperature $\left(T_{0}\right)$ and $\rho(T)$ is the resistivity at a given temperature $(T)$. When the resistivity shows a linear behavior, i.e. $\alpha$ is constant over a certain temperature range (e.g. $T_{0}$ to $T_{\max }$ ) (2) can be used, provided that $T_{0}<T<T_{\max }$ [17].

Table II shows typical values of $\rho\left(T_{0}\right)$ and $\alpha$ for various metals used in hard magnetic materials. It is observed from Table II that the reported resistivity increase with temperature [18], i.e. $\alpha>0$. It must be noted that resistivity of rare earth $\mathrm{PMs}$ such as $\mathrm{NdFeB}$ and SmCo also depends on the measuring direction as they are anisotropic materials [17].

$$
\begin{aligned}
& \alpha=\frac{1}{\rho_{0}}\left[\frac{\rho-\rho_{0}}{T-T_{0}}\right] \\
& \rho(T)=\rho\left(T_{0}\right)\left[1+\alpha\left(T-T_{0}\right)\right]
\end{aligned}
$$

TABLE II. ELECTRICAL PARAMETERS OF METALS AND MAGNETS@273K.

\begin{tabular}{|c|c|c|c|c|c|c|c|c|}
\hline \hline & Nd & Sm & Fe & Al & Ni & Co & SmCo & NdFeB \\
\hline$\rho_{0}[\Omega m]$ & 61 e-9 & 91 e-9 & $84 \mathrm{e}-9$ & $25 \mathrm{e}-9$ & $59 \mathrm{e}-9$ & $9 \mathrm{e}-9$ & $4-8 \mathrm{e}-7$ & $1.4-1.6 \mathrm{e}-6$ \\
\hline$\alpha[1 / K]$ & ND & ND & $6.5 \mathrm{e}-3$ & $4.3 \mathrm{e}-3$ & $8 \mathrm{e}-3$ & ND & ND & $2 \mathrm{e}-4$ \\
\hline
\end{tabular}

* Source: [17], [18] and [19]

**ND: No Data

\section{B. PMs resistivity vs. magnetic field}

The internal magnetic field of a PM is the result of the magnetization state of the magnet itself and of any magnetic field applied externally. Magnetization state of a PM changes due to PM temperature, (3) [2], where $\alpha_{B}$ is the magnetic field thermal coefficient (see Table I), $T$ is the temperature, $T_{0}$ is the reference temperature and $B_{0}$ is the PM flux at the reference temperature.

$$
B(T)=B_{0}\left[1+\alpha_{B}\left(T-T_{0}\right)\right]
$$

In addition to temperature, PM electrical resistivity also varies due to magnetoresistive effect [13]-[18]. $M R$ is defined as the change of the electrical resistivity of a material with its internal magnetic field [17]. MR can be modeled as (4) where $\rho_{0}$ is the resistivity of the material at the reference flux $\left(B_{0}\right)$. The resistivity can be modeled by (5), where $\beta$ is the sensitivity of the material resistance to the magnetic field. In NdFeB magnets, $\rho(B)$ decreases as $B$ increases [14]-[15], meaning that $\beta$ is negative.

$$
\begin{aligned}
& \beta=\frac{1}{\rho_{0}}\left[\frac{\rho(B)-\rho_{0}}{B(T)-B_{0}}\right] \\
& \rho(B)=\rho\left(B_{0}\right)\left[1+\beta\left(B(T)-B_{0}\right)\right]
\end{aligned}
$$

Changes of PM electrical resistivity due to an external magnetic field has already been analyzed in the literature [20]-[21], as well as the use of $M R$ for MS estimation purposes [14]-[15]. Therefore, the following analysis will 
consider only the case when PM magnetic field variations are due to PM temperature variations.

\section{Combined effect of MR and temperature on PM's electrical resistivity}

It is concluded from the previous discussion that the electrical resistivity of a PM is function of both temperature (2) and magnetic field $B(5)$. Resistivity increases with temperature but decreases with the magnetic field. A general expression of the resistivity combining both effects is given by (6). By substituting (3) into (6), (7) is obtained.

$$
\begin{aligned}
\rho(T, B) & =\rho\left(T_{0}, B_{0}\right)\left[1+\alpha\left(T-T_{0}\right)+\beta\left(B(T)-B_{0}\right)\right] \\
\rho(T, B) & =\rho\left(T_{0}, B_{0}\right)\left[1+\alpha\left(T-T_{0}\right)+\beta \alpha_{B} B_{0}\left(T-T_{0}\right)\right] \\
& =\rho\left(T_{0}, B_{0}\right)\left[1+\left(\alpha+\beta \alpha_{B} B_{0}\right)\left(T-T_{0}\right)\right]
\end{aligned}
$$

In $\mathrm{NdFeB}$ magnets the variation of the electrical resistivity with the magnetic field due to the $M R$ effect, i.e. $\beta$, is typically larger than the variation due to temperature, i.e. $\alpha,[14]$, meaning that the electrical resistivity decreases as the temperature increases. The normalized variation of the PM resistivity (7) in this case is graphically shown in Fig. 1, where $\alpha$ accounts for the direct impact of temperature on magnet resistivity and $\beta \alpha_{B} B_{0}$ account for the indirect impact of temperature on magnet resistivity due to $M R$ effect. Fully magnetized/demagnetized NdFeB magnets will be evaluated in the following sections.

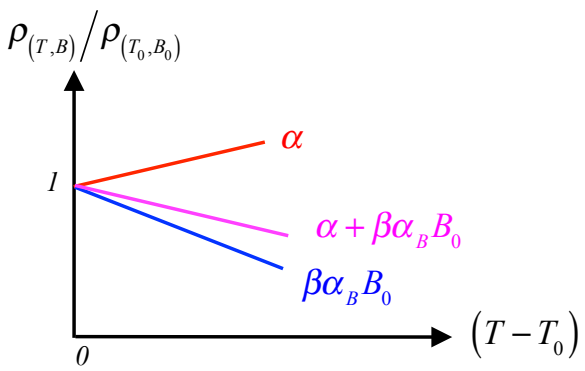

Fig. 1. Resistivity variation due to temperature and magnetic field.

\section{EXPERIMENTAL EVALUATION OF TEMPERATURE AND Field EfFects On NdFeB Magnets Resistance}

Validating the models presented in the previous section is not easy once the PMs are attached to the rotor and the PMSM is assembled [13]-[15]. Evaluation of temperature on MS on PM magnet resistivity is much easier to perform on single samples instead. Results using such simplified geometry are presented and discussed in this section.

\section{A. Experimental setup}

The system shown in Fig. 2 has been designed and built to test magnet samples. It consists of a core made of iron powder blocks, the dimensions of the central column perfectly matching with the dimensions of the magnet samples that will be evaluated.

The coil has a total number of 335 turns and is fed from a $30 \mathrm{~kW} \mathrm{H-Bridge} \mathrm{power} \mathrm{converter} \mathrm{(see} \mathrm{Fig.} \mathrm{3).} \mathrm{The}$ system enables simultaneous injection of DC currents able to change the field in the PM (a magnetic field up to $4.5 \times 10^{5} \mathrm{~A} / \mathrm{m}$ could be induced in the airgap) and $\mathrm{HF}$ currents for temperature and magnetization estimation purposes [10], [13]-[16] and [24]. In the experiments performed this paper only a high frequency current is injected. Fig. 4 shows the block diagram of the power converter control. A resonant controller is used to control the HF current $i_{h p p}^{p^{*}}$, which is used to induce controlled Eddy current in the PM from which the PM HF resistance can be estimated [14]-[15]. To measure the spatial distribution of field and temperature in the magnet, a flexibly PCB $(0.2 \mathrm{~mm}$ thickness) equipped with and array of field and temperature sensors is inserted between the core and the PM and attached to the PM. Sensors array and an example of field distribution measurement can be seen in Fig. 2c and $2 \mathrm{~d}$ respectively. Temperature sensor array is based on platinum RTD thermistors [22]. Field sensors are fed with a constant current, which minimizes the influence of temperature. Temperature effect is further compensated by software following manufacturer recommendations [23]. The field measurement system provides a measurement of both DC and HF field of the PM on its surface with a bandwidth of $300 \mathrm{kHz}$. Signals provided by temperature and field sensors are conditioned using a high precision electronics. The whole measurement system adds an airgap of $0.8 \mathrm{~mm}$ between the PM and central column of the core (see Fig. 2a).

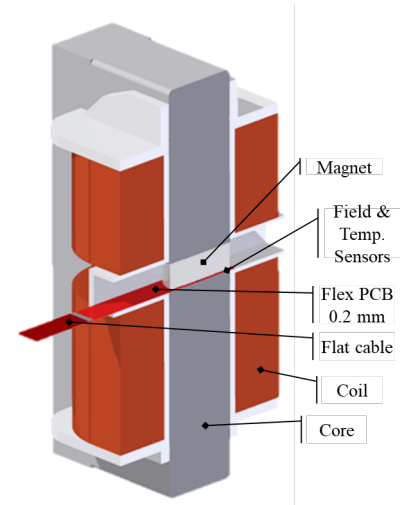

a)

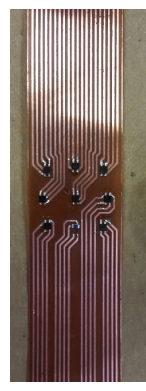

b)

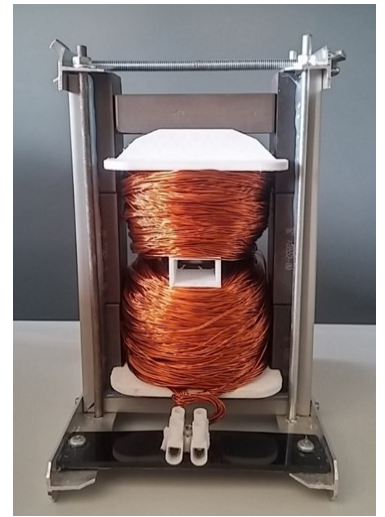

c)

Fig. 2. a) Cross-Section and b) picture of the experimental setup used for PM magnetoresitance evaluation; c) flexible PCB including field and temperature sensors and d) magnetic flux density distribution on magnet surface for a fully magnetized $\mathrm{NdFeB}$ magnet, obtained from sensors array (red dots) measurement. 


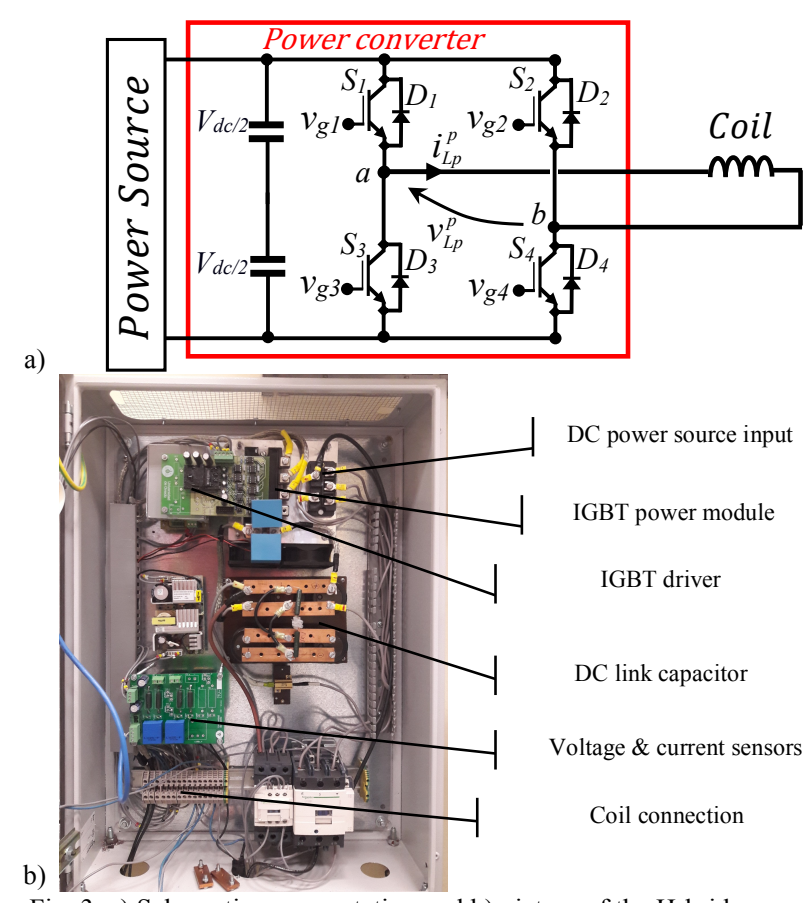

Fig. 3. a) Schematic representation and b) picture of the H-bridge power converter (b).

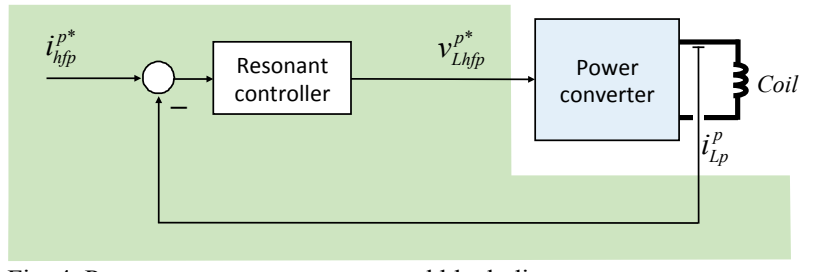

Fig. 4. Power converter current control block diagram.

\section{B. Equivalent $H F$ circuit}

The equivalent HF circuit of the experimental setup shown in Fig. 2a is shown in Fig. 5, where primary and secondary sides represent the coil and PM respectively. The HF model is described by (8) for the primary side and (9) for the secondary side; $R_{h f p}^{p}$ and $R_{h f s}^{s}$ are the coil and magnet HF resistances, $L_{h f p}^{p}$ and $L_{h f s}^{s}$ are the coil and magnet HF inductances, $\omega_{h f}$ is the frequency of the injected HF signal, $v_{h f p}^{p}$ is the coil HF voltage, $i_{h f p}^{p}$ is the coil HF current, $i_{h f s}^{s}$ magnet HF current (eddy current), $M_{p s}(10)$ is the mutual coupling between the primary and secondary and $k(10)$ is the mutual coupling coefficient. The secondary high frequency current, $i_{h f_{p}^{s}}$, can be expressed as a function of the primary current, $i_{h p p}^{P}$, mutual coupling, $M_{p s}$, magnet HF impedance, $\left(R_{h f s(T, H)}^{s}+j \omega_{h f} L_{h s s}^{s}\right)$, and frequency of the HF signal (11). By substituting (11) into (8), and dividing the resulting primary side HF voltage $\left(v_{h f p}^{p}\right)$ over the HF current $\left(i_{h f p}^{p}\right)$, the overall HF impedance seen form the coil terminals is obtained (12). It can be observed that (12) is composed by two terms, the HF impedance of the coil ( $Z_{h f_{p}}^{p}$ ) and the magnet HF impedance referred the primary side ( $\left.Z_{h f s}^{p}\right)$. The real part of the overall HF impedance $\left(R_{h f}\right)$ can be expressed as (13).

$$
\begin{aligned}
& v_{h f p}^{p}=\left(R_{h f p}^{p}+j \omega_{h f} L_{h f p}^{p}\right) i_{h f p}^{p}+j \omega_{h f} M_{p s} i_{h f s}^{s} \\
& 0=\left(R_{h f s}^{s}+j \omega_{h f} L_{h f s}^{s}\right) i_{h f s}^{s}+j \omega_{h f} M_{p s} i_{h f p}^{p}
\end{aligned}
$$

$$
\begin{aligned}
M_{p s} & =k \sqrt{L_{h s}^{s} L_{h f p}^{p}} \\
i_{h f s}^{s}= & \frac{-j \omega_{h f} M_{p s} i_{h f p}^{p}}{\left(R_{h f s}^{s}+j \omega_{h f} L_{h f s}^{s}\right)} \\
Z_{h f p}= & \frac{v_{h f p}^{p}}{i_{h f p}^{p}}= \\
= & \left(R_{h f p}^{p}+j \omega_{h f} L_{h f p}^{p}\right)+\frac{\left(\omega_{h f} M_{p s}\right)^{2}}{\left(R_{h f s}^{s}+j \omega_{h f} L_{h f s}^{s}\right)}= \\
= & Z_{h f p}^{p}+Z_{h f s}^{p}=R_{h f p}+j \omega_{h f} L_{h f p} \\
R_{h f}= & R_{h f p}^{p}+\frac{\omega_{h f}^{2} M_{p s}^{2} R_{h f s}^{s}}{\left(R_{h f s}^{s}\right)^{2}+\left(\omega_{h f} L_{h f s}^{s}\right)^{2}}=R_{h f p}^{p}+R_{h f s}^{p}
\end{aligned}
$$

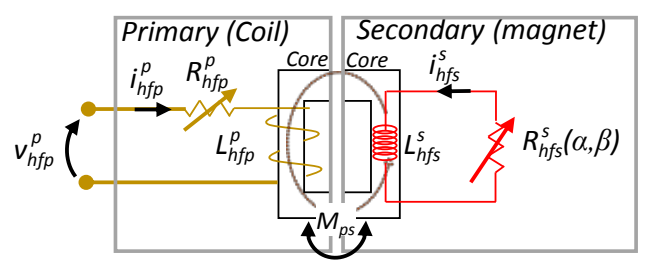

Fig. 5. Equivalent HF model of the simplified geometry.

\section{Sensitivity of $R_{h f}$ to HF circuit parameters}

This subsection analyzes the sensitivity of the (measurable) overall high frequency resistance, $R_{h f}(13)$, to the following HF circuit parameters: magnet HF resistance ( $\left.R_{h f s}^{s}\right)$, frequency of the injected signal $\left(\omega_{h f}\right)$, HF inductance of the magnet $\left(L_{h f p}^{p}\right)$, HF inductance of the coil $\left(L_{h f s}^{s}\right)$ and mutual coupling coefficient $(k)$.

Fig. 6 to Fig. 8 show the overall high frequency resistance $R_{h f}$ vs. mutual coupling coefficient $k$, injected signal frequency $\omega_{h f}$, and $L_{h f p}^{p}$ respectively. In all the cases $R_{h s s}^{s}$ varies from 0 to $3 \Omega$. It is interesting to note that in all the three cases, $R_{h f}$ does not change linearly, or even monotonically with $R_{h f s}^{s}$, i.e. an increase of $R_{h f s}^{s}$ does not necessarily produces an increase of $R_{h f}$.

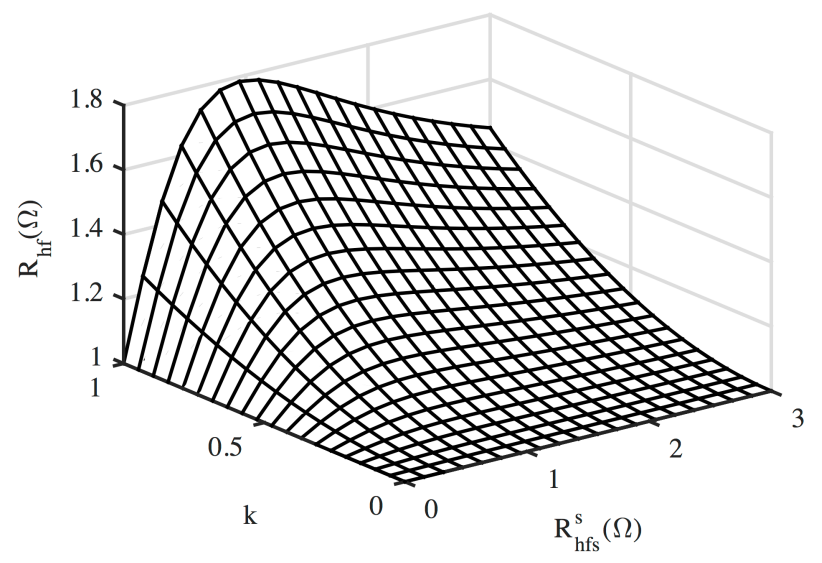

Fig. 6. Overall high frequency resistance, $R_{h f}$, vs. $R_{h f s}^{s} \& k$. $L_{h s s}^{s}=0.7 m H, L_{h f p}^{p}=1 m H, R_{h f p}^{p}=1 \Omega$ and $\omega_{h f}=2 * \mathrm{pi} * 250 \mathrm{rad} / \mathrm{s}$. 


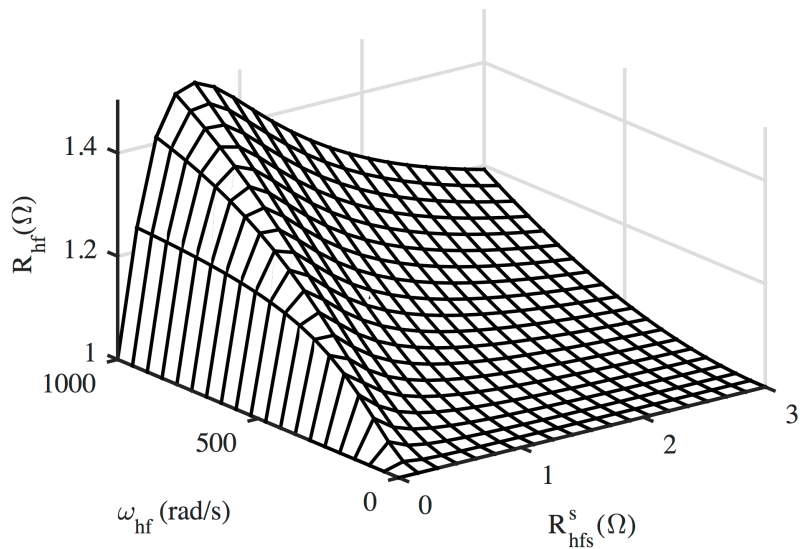

Fig. 7. Overall high frequency resistance, $R_{h f}$ vs. $R_{h f s}^{s} \& \omega_{h f}$. $L_{h f s}^{s}=0.7 \mathrm{mH}, L_{h f p}^{p}=1 \mathrm{mH}, R_{h f p}^{p}=1 \Omega$ and $k=0.75$.

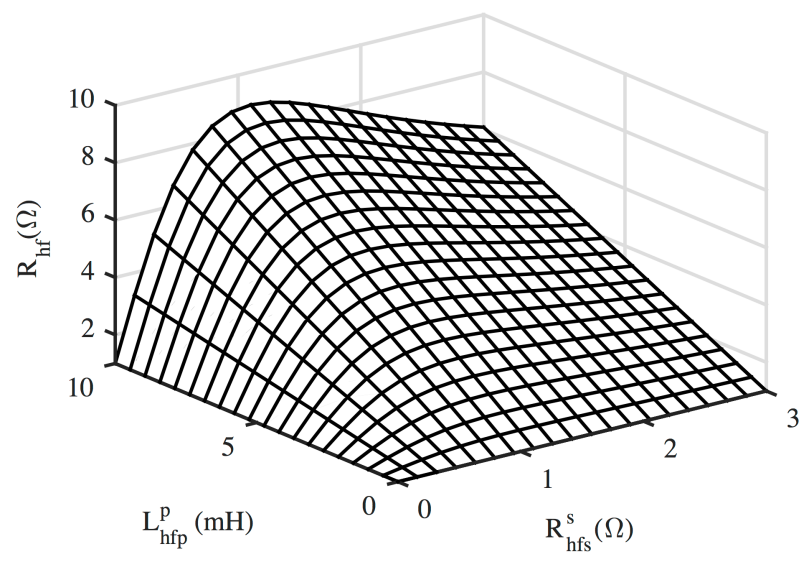

Fig. 8. Overall high frequency resistance, $R_{h f}$ vs. $R_{h f s}^{s} \& L_{h f p}^{p}$. $L_{h f s}^{s}=0.7 \mathrm{mH}, R_{h f p}^{p}=1 \Omega, k=0.75$ and $\omega_{h f}=2 * \mathrm{pi}^{*} 250 \mathrm{rad} / \mathrm{s}$.

For the case of the mutual coupling coefficient variation effect, see Fig. 6, it can be observed that the lower the mutual coupling coefficient is, the lower the overall high frequency resistance is. This result was expected: $k=0$ corresponds to the case when there is no mutual coupling between primary and secondary, therefore the overall HF resistance, $R_{h f}$, equals the coil HF resistance, $R_{h f p}^{p}$. As $k$ increases, the contribution of the primary reflected magnet HF resistance $R_{h f s}^{p}$ (see (13)) increases, $R_{h f}$ increasing too.

For the case of the frequency variation, see Fig. 7, it can be observed that the higher the frequency of the HF signal is, the higher the overall high frequency resistance is; this result is valid while the skin depth (14) is higher than the PM height [15], where $\rho$ is the PM resistivity, $\mu_{0}$ is the magnetic permeability of the air and $\mu_{r}$ is the relative magnetic permeability of the PM. For the $\mathrm{NdFeB}$ magnets used in this work, the frequency at which the skin depth equals the magnet depth is $\approx 20 \mathrm{krad} / \mathrm{s}(3.2 \mathrm{kHz})$, which is more than a full order of magnitude larger than the frequency of the signals used in this work. Skin effect can be therefore safely neglected.

$$
\delta=\sqrt{\frac{2 \rho}{\omega_{h f} \mu_{0} \mu_{r}}}
$$

For the case of $L_{h f p}^{p}$ variation effect, see Fig. 8, it can be observed that the effects are the same as those due to the variation of $k$, see Fig. 6 ; i.e. the higher $L_{h f p}^{p}$ is, the higher $R_{h f}$ is: $L_{h f p}^{p}=0$ means that there is no mutual coupling between primary and secondary, while as $L_{h f p}^{p}$ increases, the contribution of the primary reflected magnet HF resistance ( $R_{h f s}^{p}$, see (13)) increases, resulting therefore in an increase of $R_{h f}$.

Fig. 9 shows the behavior when $L_{h f s}^{s}$ varies from 0 to $10 \mathrm{mH}$ (note $L_{h f s}^{s}$ variation affects to the mutual coupling, $M_{p s}(10)$, and to the denominator $R_{h f s}^{p}$, see (13)). It can be observed that effect is opposite to the effect of $k, \omega_{h f}$ or $L_{h f p}^{p}$ , i.e. an increase of $L_{h f s}^{s}$ does not result in an increase of $R_{h f}$.

Fig. 10 shows the behavior when $L_{h f s}^{s}$ and $L_{h f p}^{p}$ vary from 0 to $10 \mathrm{mH}$ simultaneously. It is observed that $R_{h f}$ increases with $L_{h f s}^{s}$ and $L_{h f p}^{p}$. Also $R_{h f}$ does not change linearly $R_{h \mathrm{~s}}^{s}$.

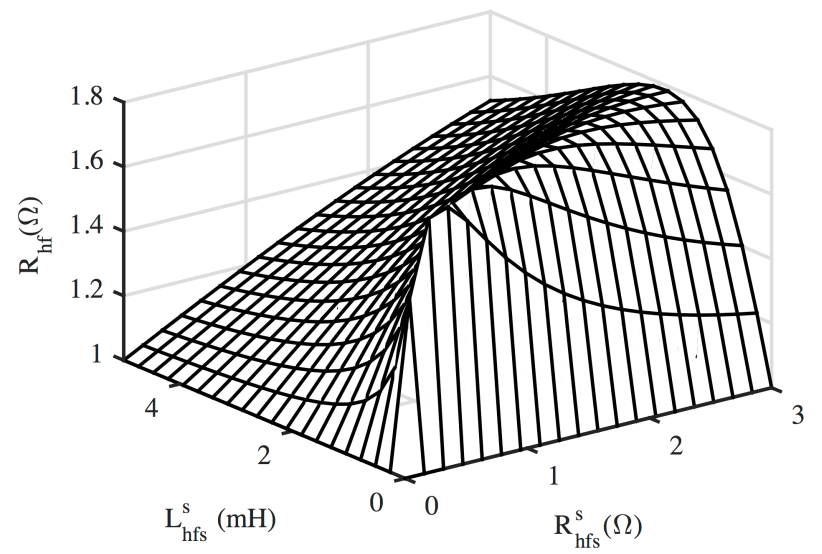

Fig. 9. Overall high frequency resistance, $R_{h f}$ vs. $R_{h f s}^{s} \& L_{h f s}^{s}$. $L_{h f p}^{p}=1 \mathrm{mH}, R_{h f p}^{p}=1 \Omega, k=0.75$ and $\omega_{h f}=2 * \mathrm{pi}^{*} 250 \mathrm{rad} / \mathrm{s}$.

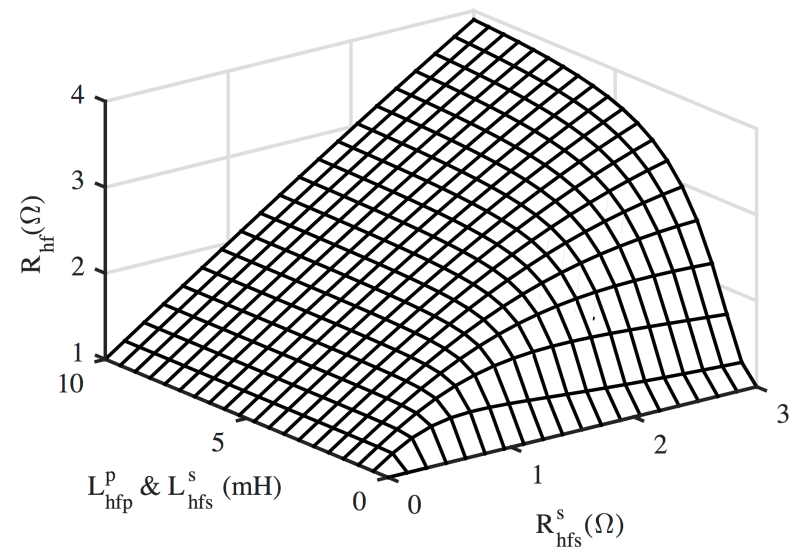

Fig. 10. Overall high frequency resistance, $R_{h f}$ vs. $R_{h f s}^{s}, L_{h f s}^{s} \& L_{h f p}^{p}$. $\omega_{h f}=2 * \mathrm{pi} * 250 \mathrm{rad} / \mathrm{s}, R_{h f p}^{p}=1 \Omega$ and $k=0.75$.

NdFeB and SmCo PMs used typically in PMSMs (have relatively high resistivity (1.4-1.6 $10^{-6} \Omega \mathrm{m}$, see Table I) compared with $\mathrm{Fe}\left(8410^{-9} \Omega \mathrm{m}\right.$, see Table II) or $\mathrm{Cu}(16.8$ $\left.10^{-9} \Omega \mathrm{m}\right)$, meaning that, generally speaking, $R_{h f s}^{s}$ is expected to have relatively high values compared with, e.g. the coil HF resistance $\left(R_{h f p}^{p}\right)$. It is also noted that $R_{h f s}^{s}$ is influenced by the PM segmentation [15], i.e. two PMs with the same shape will present higher overall electrical resistivity if the $\mathrm{PM}$ is segmented, which contributes increase $R_{h f s}^{s}$. In addition, PM HF inductance, $L_{h f s}^{s}$, is expected to be very 
low compared with the coil HF inductance, $L_{h f p}^{p}$; note that $L_{h f s}^{s}$ is determined by the path of the PM eddy currents. From the previous discussion and the analysis shown in Fig. 5 to Fig. 9, it is expected that an increase of $R_{h f s}^{s}$, e.g. due to a temperature increase will result in a decrease of the primary reflected PM HF resistance, $R_{h f}$ (see section II.C, Fig. 1 and (7)). This will be demonstrated experimentally in Sections III.C and V.

\section{Experimental results}

Fig. 11a shows the primary reflected magnet HF resistance $R_{h f s}^{p}$, for a demagnetized $\mathrm{NdFeB}$ disk (see Fig. 12) as a function of the PM temperature. PMs were demagnetized using a pulse magnetizer, details can be found in [14]-[15]. No DC current is applied in this case, meaning the there is no $M R$ effect. A HF signal of amplitude and frequency $1 \mathrm{~A}$ and $250 \mathrm{~Hz}$ respectively is injected, the $\mathrm{HF}$ resistance being estimated using the procedure described in Section III. As no DC current is being applied, the resistance variation seen in Fig. 11a is due exclusively to $\alpha$ (7). The thermal coefficient of resistivity of NdFeB PMs is positive, meaning that an increase of the PM temperature results in an increase of the PM electrical resistance $R_{h f s}^{s}$. This results in a reduction of $R_{h f s}^{p}$ (13) as discussed in Section III. Fig. 11b shows the same results as Fig. 11a but for a fully magnetized PM; $R_{h f s}^{p}$ variation is now due to the combined effect of $M R$ and temperature (7); note that $M R$ in the experimental setup core (see Fig. 2) is negligible compared to $M R$ in the PMs [14], [15] and [24]. Fig. 11c shows $R_{h f s}^{p}$ variation due to $M R$ and $\alpha$, i.e. same results as shown in Fig. $11 \mathrm{~b}$ and $R_{h f s}^{p}$ variation after decoupling the effect of $\alpha$, see Fig. 11a, i.e. variation due exclusively to $M R$ effect.

The experimental results shown in this section confirm that the estimated HF resistance of a PM is function of both temperature and $H$ due to the $M R$ effect. It is also seen from Fig. 11 that $M R$ induces larger resistivity variations than temperature.
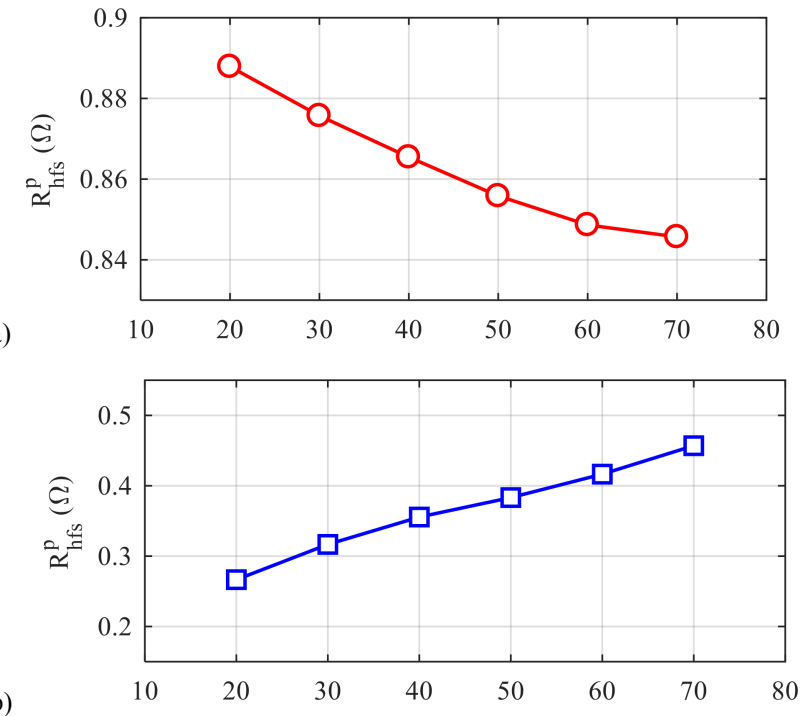

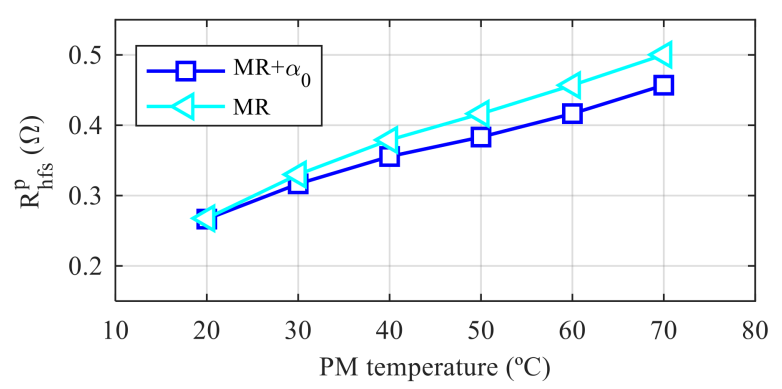

Fig. 11. PM HF resistance vs. temperatures a) for a demagnetized PM, b) fully magnetized PM, and c) fully magnetized PM after decoupling the effect of $\alpha$ effect. $\omega_{\mathrm{hf}}=2 \pi 250 \mathrm{rad} / \mathrm{s}, \mathrm{I}_{\mathrm{hf}}=1 \mathrm{~A}(0.05 \mathrm{pu})$.

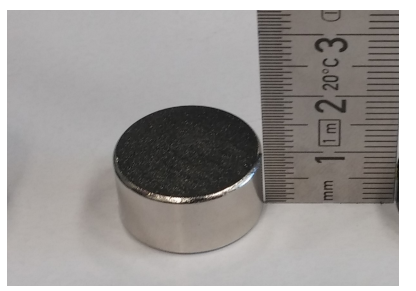

Fig. 12. Magnet sample of $\varnothing 20 \times 10 \mathrm{~mm}$ for the experimental setup.

\section{PM HIGH FREQUENCY RESISTANCE ESTIMATION IN PMSMS}

\begin{tabular}{|c|c|}
\hline \multicolumn{2}{|c|}{ Table III } \\
\hline $\begin{array}{c}\text { Variables in the experimental } \\
\text { setup HF model }\end{array}$ & $\begin{array}{c}\text { Variables in the PMSM HF } \\
\text { model [9] }\end{array}$ \\
\hline $\begin{array}{l}v_{h f p}^{p}: \text { primary high frequency } \\
\text { voltage }\end{array}$ & $\begin{array}{l}v_{d h f}^{r}: \text { stator } d \text {-axis high frequency } \\
\text { voltage }\end{array}$ \\
\hline $\begin{array}{l}i_{h f p}^{p}: \text { primary high frequency } \\
\text { current }\end{array}$ & $\begin{array}{l}i_{d h f}^{r}: \text { stator } d \text {-axis high frequency } \\
\text { current }\end{array}$ \\
\hline $\begin{array}{l}L_{h f p}^{p}: \text { primary high frequency self- } \\
\text { inductance }\end{array}$ & $\begin{array}{l}L_{d h f}: \quad d \text {-axis high frequency } \\
\text { inductance }\end{array}$ \\
\hline $\begin{array}{l}R_{h f p}^{p}: \text { primary high frequency } \\
\text { resistance }\end{array}$ & $\begin{array}{l}R_{d s h f}: \text { stator } d \text {-axis high frequency } \\
\text { resistance }\end{array}$ \\
\hline $\begin{array}{l}M_{p s}: \text { mutual coupling between the } \\
\text { primary and the secondary }\end{array}$ & $\begin{array}{l}M_{D d}: \text { mutual coupling between } \\
\text { stator } d \text {-axis and rotor } d \text {-axis }\end{array}$ \\
\hline $\begin{array}{l}L_{h f s}^{s}: \text { secondary high frequency } \\
\text { self-inductance }\end{array}$ & $\begin{array}{l}L_{d r h f}: \text { magnet high frequency self- } \\
\text { inductance }\end{array}$ \\
\hline $\begin{array}{l}R_{h f s}^{s}: \text { secondary high frequency } \\
\text { resistance }\end{array}$ & $\begin{array}{l}R_{d r h f}: \text { magnet high frequency } \\
\text { resistance }\end{array}$ \\
\hline
\end{tabular}

Magnet samples have been used in the previous section to study the effect of temperature and $M R$ on PM resistance. However, the final target of the proposed methods is their use in inverter fed PMSMs. It is therefore necessary to establish the connection between the system described in Sections III-IV and the PMSM. Fig. 13 shows a schematic representation of the PMSM that will be used for the experimental verification and the equivalent HF circuit of $d$ axis of the machine. Comparing the equivalent circuits of the experimental setup in Fig. 5 and the PMSM in Fig. 13, the similarities between both systems become evident. Table III shows the equivalence between the experimental setup, (8)-(13), and the HF model of a PMSM [9]. Consequently, it is realistic to assume that the results and conclusions obtained for the experimental platform can be extended to the PMSM case. 

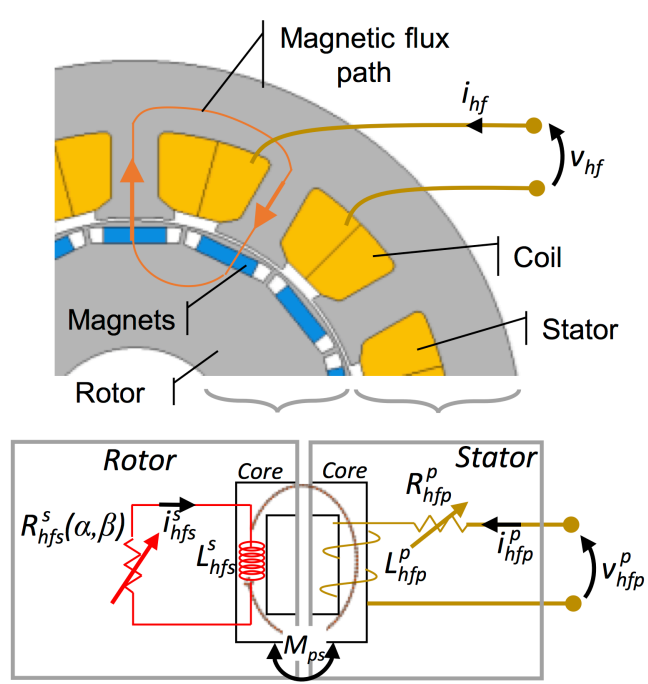

Fig. 13. Top- Schematic representation of a PMSM; bottom - Simplified equivalent $d$-axis high frequency circuit of an IPMSM for pulsating high frequency signal.

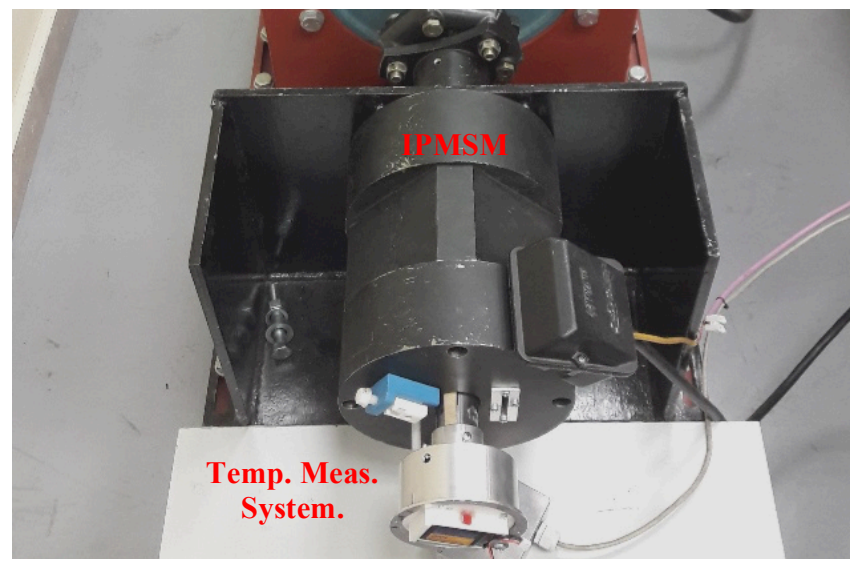

Fig. 14. IPMSM with the wireless PM temperature measurement system.

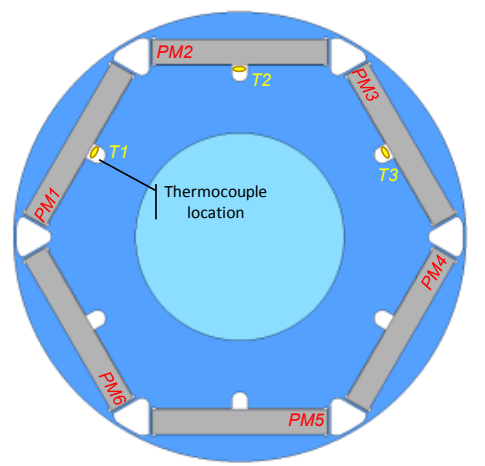

Fig. 15. Cross-section of the rotor and thermocouples location.

Several options have been reported for the injection of a HF signal in PMSM for PM temperature and magnetization state estimation purposes [10], [11], [13] and [14]. Among these pulsating high-frequency current has shown to provide the most appealing properties [10]. The overall HF impedance and resistance seen form the stator terminals of the machine can therefore be obtained from (12) and (13), respectively. Note that since the HF current is controlled to be aligned with rotor $d$-axis, the rotor path seen by the high-frequency flux is independent of rotor position. Consequently mutual coupling between stator $d$ - axis and rotor d-axis $\left(M_{D d}\right)$ is not affected by rotor position [8], [10], [12] and [13].

\section{EXPERIMENTAL RESULTS WITH AN IPMSM}

TABLE III. IPMSM PARAMETERS
\begin{tabular}{|l|l|l|l|l|l|l|}
\hline PATED $_{\text {RATED }}$ & $\mathrm{V}_{\text {RATED }}$ & $\mathrm{I}_{\text {RATED }}$ & $\omega_{\mathrm{r}}$ & Poles & Slots & Magnet \\
\hline $7.5 \mathrm{~kW}$ & $350 \mathrm{~V}$ & $14 \mathrm{~A}$ & $1800 \mathrm{rpm}$ & 6 & 36 & N-42SH \\
\hline
\end{tabular}

The same experiments performed with the simplified geometry described in section IV have been carried using an PMSM. Details of the machine can be seen in Fig. 14 and Fig. 15; machine parameters being shown in Table IV. The rotor of the machine has a special design, assembling/disassembling of the PMs being possible by means of an aluminum guide that has been installed in one end shield. Three thermocouples attached to three magnets (see Fig. 15) are used to measure PMs temperatures. The thermocouple wires are taken out throughout a slot in the shaft and connected to a measurement and wireless transmission device. The schematic representation of the temperature measurement wireless device is shown in Fig. 16. The device allows online PM temperature measurement, i.e. while the machine is running, at a sampling rate of $1 \mathrm{~Hz}$. For all experimental results shown in this section the amplitude and frequency of the HF signal are $1 \mathrm{~A}$ and 250 $\mathrm{Hz}$ respectively. The machine rotates at $\omega_{\mathrm{r}}=100 \mathrm{rad} / \mathrm{s}$.

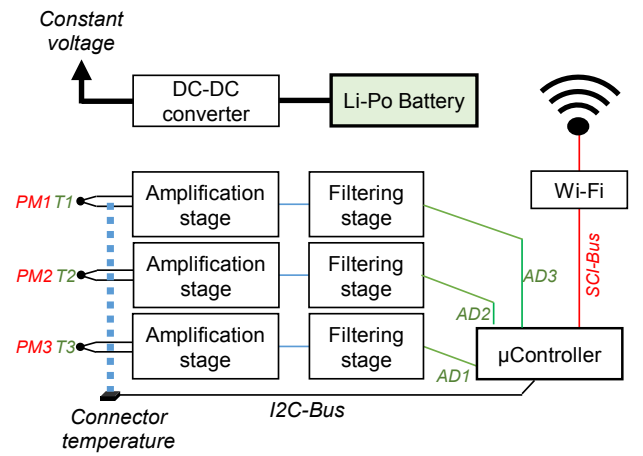

Fig. 16. Schematic representation of the PM temperature measurement system.

a)

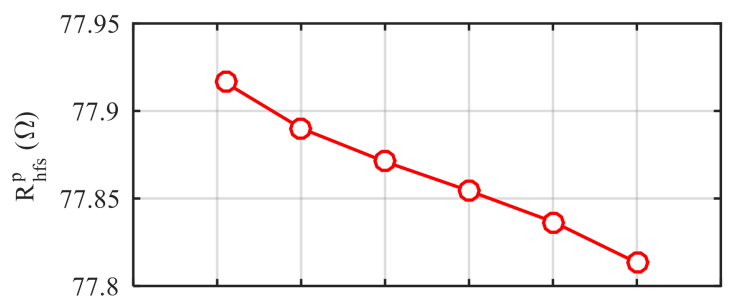

b)

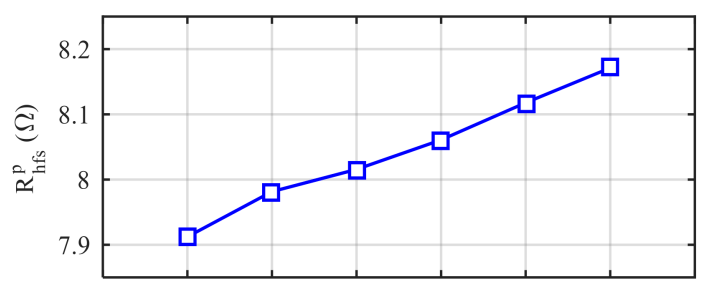




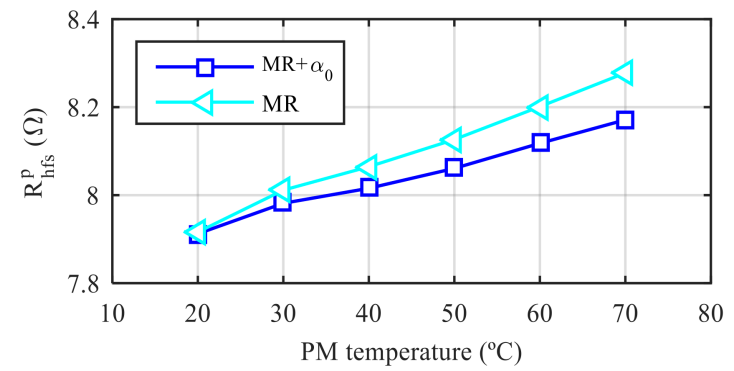

Fig. 17. PM HF resistance of IPMSM vs. temperatures a) for a demagnetized PM, b) fully magnetized PM, and c) fully magnetized PM after decoupling the effect of $\alpha . \mathrm{I}_{\mathrm{dhf}}=1 \mathrm{~A}, \omega_{\mathrm{hf}}=2 \pi 250 \mathrm{rad} / \mathrm{s}, \omega_{\mathrm{r}}=100 \mathrm{rad} / \mathrm{s}$.

Fig. 17a shows the stator reflected PM HF resistance, $R_{h s s}^{p}$ (13) of the PMSM, when all 6 PMs (see Fig. 15) are fully demagnetized as PM temperature changes (all PMs having the same temperature). To obtain $R_{h f s}^{p}$, it is assumed that the core losses are negligible compared with the PM losses; $M R$ effect in the core being therefore negligible compared with $M R$ effect in PMs [14]-[15]. This is realistic considering that the core is laminated and the magnets are not. The machine is heated up using fundamental current, once PM reaches $70{ }^{\circ} \mathrm{C}$, excitation is removed and $R_{h f s}^{p}$ is estimated by injecting a HF signal while the machine is cooling down. Since PMs are demagnetized and there is no external field applied, no $M R$ effect exists, the resistance variation seen in Fig. 17a being therefore due to $\alpha$ exclusively. Consistently with Fig. 11a, $R_{h f s}^{p}$ decreases as the temperature increases. Fig. $17 \mathrm{~b}$ shows $R_{h f \mathrm{~s}}^{p}$ of a fully magnetized PM as the PM temperature changes. The resistance variation seen in this figure is due to the combined effect of $M R$ and $\alpha$. Fig. 17c shows $R_{h f s}^{p}$ variation due to $M R$ and $\alpha$, i.e. same results as shown in Fig. $17 \mathrm{~b}$ and $R_{h f s}^{p}$ variation after decoupling the effect of $\alpha$, see Fig. 17a, i.e. variation exclusively due to $M R$ effect. It is observed that there is a good agreement between the results for the PMSM in Fig. 17 for the simplified geometry in Fig. 11. The experimental results shown in this section confirm that the estimated HF resistance of a PMSM is function of both temperature and MS of the PMs due to the $M R$ effect. Consistently with the experimental results shown in Fig. 11, $M R$ induces larger resistivity variations than $\alpha$.

\section{CONCLUSIONS}

This paper studies the effects of PM temperature and MS on PM resistivity. Proper understanding of these effects is required to improve the accuracy and robustness of temperature and MS estimation methods using PM resistance. It has been shown that temperature and MS affect to the PM high frequency resistance. Thermal coefficient and MS coefficient have opposite effect on the PM electrical resistivity. Experimental verification has been performed both on magnet samples and on a PMSM. Ongoing research is targeted toward the development of models able to separate temperature and MS effects.

\section{ACKNOWLEDGMENT}

The authors would like to acknowledge BOMATEC for the support and providing magnet samples.

\section{REFERENCES}

[1] N. Bianchi and T. M. Jahns, "Design, analysis and control of interior PM synchronous machines," Tutorial Course Notes, Seattle, Oct. 5, 2004.

[2] J. F. Gieras and M. Wing, "Permanent magnet motor technology: design and application". Second edition 2002.

[3] C. Deak, A. Binder, B. Funieru and M. Mirzaei, "Extended field weakening and overloading of high-torque density permanent magnet motors," 2009 IEEE Energy Conversion Congress and Exposition, San Jose, CA, 2009, pp. 2347-2353. doi: 10.1109/ECCE.2009.5316366

[4] M. Ganchev, C. Kral and T. M. Wolbank, "Compensation of Speed Dependence in Sensorless Rotor Temperature Estimation for Permanent-Magnet Synchronous Motor," in IEEE Transactions on Industry Applications, vol. 49, no. 6, pp. 2487-2495, Nov.-Dec. 2013.

[5] D. Fernandez, D. Reigosa, T. Tanimoto, T. Kato and F. Briz, "Wireless permanent magnet temperature \& field distribution measurement system for IPMSMs," 2015 IEEE Energy Conversion Congress and Exposition (ECCE), Montreal, QC, 2015, pp. 39964003. doi: 10.1109/ECCE.2015.7310224

[6] Bomatec Magnets. Product Catalog. Accessed: June 2017. Available online at: http://www.bomatec.ch/en/products/

[7] A. M. EL-Refaie, N. C. Harris, T. M. Jahns and K. M. Rahman, "Thermal analysis of multibarrier interior PM synchronous Machine using lumped parameter model," in IEEE Transactions on Energy Conversion, vol. 19, no. 2, pp. 303-309, June 2004.doi: 10.1109/TEC.2004.827011

[8] C. Kral, A. Haumer and S. B. Lee, "A Practical Thermal Model for the Estimation of Permanent Magnet and Stator Winding Temperatures," in IEEE Transactions on Power Electronics, vol. 29, no. 1, pp. 455-464, Jan. 2014. doi: 10.1109/TPEL.2013.2253128

[9] K. Liu and Z. Q. Zhu, "Online Estimation of the Rotor Flux Linkage and Voltage-Source Inverter Nonlinearity in Permanent Magnet Synchronous Machine Drives," in IEEE Transactions on Power Electronics, vol. 29, no. 1, pp. 418-427, Jan. 2014. doi:10.1109/TPEL.2013.2252024

[10] D. D. Reigosa, D. Fernandez, H. Yoshida, T. Kato and F. Briz, "Permanent-Magnet Temperature Estimation in PMSMs Using Pulsating High-Frequency Current Injection," in IEEE Transactions on Industry Applications, vol. 51, no. 4, pp. 3159-3168, July-Aug. 2015. doi: 10.1109/TIA.2015.2404922

[11] D. D. Reigosa, F. Briz, P. Garcia, J. M. Guerrero and M. W. Degner, "Magnet Temperature Estimation in Surface PM Machines Using High-Frequency Signal Injection," in IEEE Transactions on Industry Applications, vol. 46, no. 4, pp. 1468-1475, July-Aug. 2010. doi: 10.1109/TIA.2010.2049816

[12] M. Ganchev, C. Kral, H. Oberguggenberger and T. Wolbank, "Sensorless rotor temperature estimation of permanent magnet synchronous motor," IECON 2011 - 37th Annual Conference of the IEEE Industrial Electronics Society, Melbourne, VIC, 2011, pp. 2018-2023. doi: 10.1109/IECON.2011.6119449

[13] D. Reigosa, D. Fernandez, T. Tanimoto, T. Kato and F. Briz, "Comparative Analysis of BEMF and Pulsating High Frequency Current Injection Methods for PM Temperature Estimation in PMSMs," in IEEE Transactions on Power Electronics , vol.PP, no.99, pp.1-1 doi: 10.1109/TPEL.2016.2592478

[14] D. Díaz Reigosa, D. Fernandez, Z. Q. Zhu and F. Briz, "PMSM Magnetization State Estimation Based on Stator-Reflected PM Resistance Using High-Frequency Signal Injection," in IEEE Transactions on Industry Applications, vol. 51, no. 5, pp. 3800-3810, Sept.-Oct.

2015. doi: 10.1109/TIA.2015.2437975

[15] D. Fernandez, D. D. Reigosa, J. M. Guerrero, Z. Q. Zhu and F. Briz, "Permanent-Magnet Magnetization State Estimation Using HighFrequency Signal Injection," in IEEE Transactions on Industry Applications, vol. 52, no. 4, pp. 2930-2940, July-Aug. 2016. doi: 10.1109/TIA.2016.2541616

[16] D. F. Alonso, D. Reigosa, M. Martinez, J. Guerrero and F. Briz, "Influence of magnetoresistance and temperature on permanent 
magnet condition estimation methods using high frequency signal injection," 2017 IEEE Energy Conversion Congress and Exposition (ECCE), Cincinnati, OH, USA, 2017, pp. 3201-3207. doi: 10.1109/ECCE.2017.8096581

[17] Kasap, S. O. (2006). Principles of Electronic Materials and Devices (Third ed.). Mc-Graw Hill. p. 126.

[18] S. Ruoho, M. Haavisto, E. Takala, T. Santa-Nokki and M. Paju, "Temperature Dependence of Resistivity of Sintered Rare-Earth Permanent-Magnet Materials," in IEEE Transactions on Magnetics, vol. 46, no. 1, pp. 15-20, Jan. 2010. doi: 10.1109/TMAG.2009.2027815

[19] EMAGNETS. NdFeB electrical characterystics, 2017. Available online at:http://emagnetsuk.com/neodymium_magnets/characteristics.aspx, Acceessed 12-Jan-2017.

[20] B. Idzikowski, M. Wolf, A. Handstein, K. Nenkov, F. Stobieski, and K.-H. Muller, "Inverse giant magnetoresistance in granular $\mathrm{Nd} / \mathrm{sub}$
2/Fe/sub 14/ B a-Fe,” in Dig. INTERMAG Magn. Conf., Oct. 1997, doi: 10.1109/INTMAG.1997.597496.

[21] B. Idzikowski, M. Wolf, A. Handstein, K. Nenkov, F. Stobieski, and K.-H. Muller, "Inverse giant magnetoresistance in granular $\mathrm{Nd} 2 \mathrm{Fe} 14$ B/ $\alpha$-Fe,” IEEE Trans. Magn., vol. 33, no. 5, pp. 3559-3561, Sep. 1997.

[22] Vishay - Sensors, Temperature, Thin Film RTD "Platinum SMD Flat Chip Temperature Sensor," PTS AT, Jun. 3, 2017. [Online]. Available: http://www.vishay.com/sensors/sensorstemperature/platinum.

[23] ChenYang - sensors and measurements, "Selection guide of hall effect sensor elements/ICs," CYSJ106C, Mar. 27, 2017. [Online]. Available: http://www.hallsensors.de

[24] D. Fernandez, D. Reigosa, J. M. Guerrero, Z.Q. Zhu, C. Suarez and F. Briz, "Influence of PM Coating on PM Magnetization State Estimation Methods Based on Magnetoresistive Effect" in IEEE Transactions on Industry Applications, vol. 53 (accepted publication pending), 2018. doi: 10.1109/TIA.2018.2797883 